\title{
Avant-propos de la traduction française
}

Dans cette traduction, la formulation en (en italique) est utilisée tout au long de cet ouvrage comme abréviation pour le ligand éthylènediamine (ou 1,2-diaminométhane). Cela ne relève que du souci d'éviter la confusion avec l'adverbe et la préposition : en, largement utilisés en français. II ne s'agit en aucun cas d'un formalisme d'écriture chimique.

Cette traduction a bénéficié de la relecture attentive et des corrections avisées d'Isabelle Michaud-Soret, Directrice de Recherche au CNRS, responsable de l'équipe Biomet du Laboratoire de Chimie et Biochimie des Métaux. Je l'en remercie et, comme l'indique l'auteur, d'éventuelles erreurs non relevées seraient à mettre à mon unique débit.

Jacques Covès

Grenoble, mars 2017 
